\title{
Association between maternal antenatal depression and infant development: a hospital-based prospective cohort study
}

\author{
Yuko Otake - Sonomi Nakajima $\cdot$ Akiko Uno • \\ Shizue Kato $\cdot$ Seiko Sasaki $\cdot$ Eiji Yoshioka $\cdot$ \\ Tamiko Ikeno $\cdot$ Reiko Kishi
}

Received: 1 April 2013/Accepted: 9 July 2013/Published online: 4 August 2013

(C) The Japanese Society for Hygiene 2013

\begin{abstract}
Objective To examine the association between antenatal depression and infant development after controlling for confounding factors.

Methods A hospital-based prospective cohort study (Hokkaido Study on Environment and Children's Health) was conducted between July 2002 and October 2005 in Sapporo, Japan. Of 309 mothers who delivered at Sapporo Toho Hospital during the study period and who agreed with the clinical assessment of depression, 154 mother-infant pairs were eligible for analysis. Antenatal depression was assessed between the second and third trimesters using the Edinburgh Postnatal Depression Scale (EPDS), and infant development was assessed at 6 months by the Bayley Scales of Infant Development II (BSID-II). Data on potential confounders, including socioeconomic status, birth complications, postnatal depression and child care
\end{abstract}

Y. Otake $\cdot$ T. Ikeno $\cdot$ R. Kishi $(\bowtie)$

Centre for Environmental and Health Science, Hokkaido

University, North 12, West 7, Kita-ku, Sapporo 060-0812, Japan

e-mail: rkishi@med.hokudai.ac.jp

Y. Otake

e-mail: yukotakey@gmail.com

S. Nakajima

Department of Occupational Therapy, Sapporo Medical

University School of Sciences, Sapporo, Japan

A. Uno $\cdot$ S. Kato $\cdot$ S. Sasaki

Department of Public Health, Hokkaido University Graduate

School of Medicine, Sapporo, Japan

E. Yoshioka

Division of Community Medicine and Epidemiology,

Department of Health Science, Asahikawa Medical University, Asahikawa, Japan environment, were obtained from medical records and selfadministered questionnaires. Univariable and multivariable analyses were conducted in which the EPDS score was entered as an independent variable and the BSID-II scores as a dependent variable, adjusting for confounders.

Results Although the antenatal EPDS score tended to be related to the BSID-II score in the univariable analysis, this correlation was lost in the multivariable analysis. However, based on a series of linear regression analyses, antenatal depression was found to be significantly related to shorter gestational age $(\beta=-0.25,95 \%$ confidence interval (CI) $[-1.20,-0.17])$, and shorter gestational age was significantly related to a lower BSID-II (mental development) score $(\beta=0.23,95 \%$ CI $[0.00,0.00])$.

Conclusions Gestational age is an important confounder in the association between maternal antenatal depression and infant development. A delay in infant development may be related to a shorter gestational period caused by maternal depression during pregnancy.

Keywords Maternal depression · Pregnancy · Infant development $\cdot$ Gestational age $\cdot$ Cohort study

\section{Introduction}

There is increasing recognition for the relationship between maternal psychological distress during pregnancy, such as maternal antenatal stress, anxiety, and depression and infant development, and various studies have been conducted using animal models, human physiology and epidemiology. Results from animal experiments suggest that maternal stress during pregnancy is associated with alterations in brain function and behaviour in infants. The fetuses of mothers who experience stress show alterations 
in activation of the hypothalamic-pituitary-adrenal (HPA) axis and in brain function compared to fetuses of nonstressed mothers $[1,2]$. According to a review of animal experiments, infants born to rodent mothers exposed to antenatal stress demonstrate more problems in learning behaviour than infants of non-stressed mothers [3].

Physiological mechanisms in humans have been proposed by several researchers [2-4]. Antenatal anxiety appears to raise uterine artery resistance, which can influence fetal development and infant birth weight [3]. The psychological status of pregnant women is known to alter the intrauterine environment and function of the fetal HPA axis, which in turn influences longitudinal behavioural and psychological development of infants after birth [2, 4].

Given these observations, epidemiological studies on human populations have been carried out in recent years [5-7]. For example, one report from a large cohort study, the Avon longitudinal study of parents and children (ALSPAC) [6], showed that antenatal depression influences child development independently of postnatal depression. The ALSPAC study also found that anxiety during pregnancy continues to affect child development 4 years after birth [7]. Another study exploring mothers who were pregnant at the time of a tornado disaster in Canada revealed the impact of strong objective stress during pregnancy on the IQ and language capability of infants [5]. Even though exposures to maternal antenatal depression, stress and anxiety are believed to be correlated to one another, there has been less investigation into the effects of antenatal depression than other maternal psychological factors [7-9]. Our study therefore focused on the relation of antenatal depression and infant development.

Previous studies examining antenatal depression and infant development are characterised by two important limitations: contradictory findings and the omission of confounding factors related to child rearing. Although some studies have insisted that antenatal depression is related to lower infant development scores that indicate a developmental delay $[6,10]$, others have related antenatal depression to higher performance in infant development tests [11] or have shown no correlation with infant development [12]. The ALSPAC study [6] and the study by DiPietro et al. [11] demonstrated contradictory effects of antenatal depression, despite the fact that both studies were conducted using prospective birth cohorts and applied globally standardised measures, including the Edinburgh Postnatal Depression Scale (EPDS) or the Center for Epidemiologic Studies Depression Scale for assessing maternal depression, and the Denver Developmental Screening Test (DDST) or the Bayley Scales of Infant Development II (BSID-II) for assessing infant development. Although the study population of the ALSPAC was large (9,244 women), the study had a number of limitations, such as the small number of depressed mothers and the use of maternal self-reporting to assess infant development [6]. DiPietro et al. [11] used structured assessment to avoid the problem of self-reporting; however, the number of participants was much smaller (94), which limited the study's statistical power.

In addition, confounding factors were not sufficiently controlled for in these earlier studies. In these studies, researchers controlled for diverse maternal and infant factors, including antenatal and postnatal maternal psychological distress, maternal smoking during pregnancy, maternal age, maternal educational level, infant birth weight and gender and infant age at the time of developmental assessment [6, 10-12]. However, they did not consider differences in the rearing attitude of the parents or in the home environment. Infant development is strongly influenced by interactions between the infant and the stimuli surrounding them. For example, mother-child interactions and maltreatment are well-known factors directly affecting infant development [13-15]. When infants do not obtain appropriate stimulation from their caregivers, developmental problems typically result. Therefore, examination of child rearing factors as confounders during the postnatal period is necessary.

Given these two principal limitations to previous studies, the purpose of our study was to examine the association between antenatal depression and infant development while controlling for child care factors in addition to other confounders considered in previous studies.

\section{Methods}

Study design and population

A prospective cohort study was carried out between July 2002 and October 2005 at the Sapporo Toho Hospital in Hokkaido, Japan (Hokkaido Study on Environment and Children's Health). Pregnant women who were at 23-35 weeks of gestation during a routine gynaecological check-up in this study period were recruited to the study. All participants were native Japanese and residents of Sapporo or the surrounding area. Of 1,796 potentially eligible women, 514 agreed to participate (Fig. 1; $30 \%$ participation rate). Of all the potential participants we approached, women who had registered for the Japanese Cord Blood Bank (22\% of those who were approached) and those who delivered at another hospital ( $3 \%$ of those who were approached) were excluded from the study cohort. Some of the women we approached were not interested in our study, and some were unable or unwilling to participate.

Assessment of depression during pregnancy was conducted between October 2002 and April 2004 as a nested 


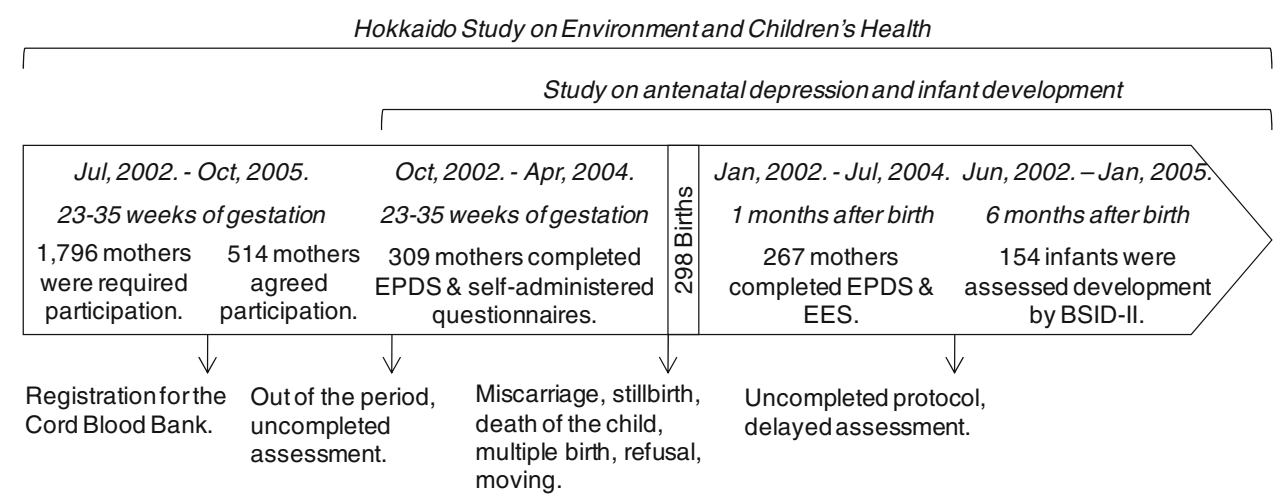

Fig. 1 Selection process for participant eligibility in Hokkaido Study. The prospective cohort study was performed between July 2002 and October 2005 at Toho Hospital (Hokkaido Study on Environment and Children's Health). A total of 1,796 pregnant women were invited to participate in the study during a routine gynaecological checkup and 514 women agreed. Women who were registered for the Cord Blood Bank were not eligible to participate. Assessment of depression during pregnancy was conducted between October 2002 and April 2004; 309 women were involved in the assessment and completed questionnaires (60\% of the initial cohort). Pregnant women who were outside of the study period or who failed

cohort study within the Hokkaido Study. Pregnant women who were recruited to the Hokkaido Study during this period were assessed for antenatal depression, and 309 women completed questionnaires (60\% of initial cohort). Postnatal depression was assessed in 267 mothers between 1 and 4 months after delivery, and infant development was evaluated in 154 mother-infant pairs during the period from 5 months and 16 days up to 6 months and 15 days after birth (50\% follow-up rate). Excluded participants were those who did not complete the protocol due to miscarriage, stillbirth, multiple birth, relocation, death of the infant, or voluntary withdrawal from the study. Statistical analysis was conducted for 154 mother-infant pairs.

\section{Exposure measure}

The EPDS was used to evaluate the incidence of antenatal depression, and pregnant women at 23-35 weeks of gestation were required to complete the EPDS questionnaire at recruitment. We assessed maternal depression between the second and third trimesters since this is the period of fetal development, and previous studies also assessed maternal psychological distress during this period $[6,11,12]$. The EPDS is a widely used self-rating questionnaire [18] and has been used during the antenatal period even though originally developed as a screening tool for maternal depression following childbirth [6]. Because the validity and reliability of the EPDS in Japanese women has been established [19], it has been commonly used for the screening of postnatal depression in Japanese community to complete the assessment were eliminated from the cohort. Postnatal depression was assessed in 267 mothers between 1 and 4 months post-delivery, and infant development was assessed in 154 motherinfant pairs during the period from 5 months and 16 days to 6 months and 15 days after birth (50\% follow-up rate). Excluded participants were those who did not complete the protocol due to miscarriage, stillbirth, multiple birth, relocation, death of the infant or voluntary withdrawal from the study. Statistical analysis was conducted for 154 mother-infant pairs. EPDS Edinburgh Postnatal Depression Scale, EES evaluation of Environmental Stimulation, BSID-II Bayley Scales of Infant Development II

settings. The EPDS comprises ten questions evaluating depressive symptoms. Women rate their feelings over the previous 7 days using a score from 0 to 30 . The standardised cut-off of 8/9 was applied for Japanese women (with a score of $\geq 9$ considered to indicate depression [19]) because Japanese women tend to score lower than Englishspeaking women for whom the suggested cut-off is $12 / 13$ [18].

\section{Outcome measure}

Infant development was assessed at 6 months after birth using the BSID-II [20], one of the most widely used and validated assessment tools for preschool children. Because the BSID-II is not standardised in Japan, we translated a BSID-II manual in consultation with a manual for BSID which used in the Hokkaido Study [21]. The validity of the BSID-II for Japanese infants was previously evaluated by referring to the DDST [22], and it was used in one of the Hokkaido Study analyses to assess the effects of antenatal exposure to polychlorinated biphenyls and dioxins on infant development [21]. The BSID-II consists of a mental development index (MDI) for assessing cognitive, language and personal/social development and a psychomotor development index (PDI) for assessing fine and gross motor development. MDI and PDI scores range from 50 to 150. In the USA, a mean value of 100 has been established as the cut-off point for each index. However, because the cut-off for Japanese infants requires further investigation [23], we used the total PDI and MDI scores in our study. 
For the assessment, infants were brought to the community centre in Sapporo where they were tested in a quiet, private room in the presence of one or both parents. Each evaluation was performed by one of three occupational therapists with clinical experience in the field of developmental disabilities. The examiners were unaware of the antenatal EPDS scores of the mothers. In all cases, the therapist who performed the examination calculated the infant's score which was then double-checked by the other two examiners based on a video recording of the examination. The final score was decided through discussion and agreement by all three examiners.

\section{Confounder measures}

\section{Characteristics of participants}

Participants completed a self-administered questionnaire at the time of recruitment (between 23 and 35 weeks of gestation) which included questions on maternal smoking, caffeine intake, alcohol intake, drug use, working status during pregnancy, educational level of both parents and household income. Information on the anamnesis of thyroid disease and mental illness was also obtained through the questionnaire. Maternal smoking was categorised as either "no" (non-smokers who did not smoke throughout pregnancy or who quit smoking during the first trimester) or "yes" (smokers who continued to smoke during pregnancy, including women who quit after the first trimester). Modified self-administered questionnaires described by Nagata et al. [16, 17] were used to estimate caffeine and alcohol intake. Information on drug use and anamnesis of parents included medication taken at the time of study and a complete disease history. Perinatal information was obtained from obstetrical records and included age of parents at childbirth, pregnancy complications, gestational age, and infant sex, parity, disease, birth weight and birth size (length, head circumference, chest circumference). Information on maternal working status at 6 months after delivery was obtained using the self-reported questionnaire at the 6-month infant assessment.

\section{Maternal psychological status \\ before and during pregnancy}

At the time of recruitment, pregnant women were also asked to complete self-rating questionnaires which were originally developed in this study to determine psychological status before and during pregnancy. Women answered of "yes" or "no" to questions on (1) stressful life events during the year before pregnancy ("Have you experienced stressful life events during the past year?"); (2) maternal neuroses, including past depressive symptoms
("Have you felt continuous depression or unhappiness every day for more than 2 weeks before pregnancy?"), worrying ("Do you think of yourself as a worrier?") and obsessiveness ("Do you think of yourself as obsessive?"); (3) readiness for pregnancy, including planned pregnancy ("Did you plan to be pregnant?") as well as wanted pregnancy ("Did you want to be pregnant?").

\section{Maternal postnatal depression}

The EPDS was used to evaluate postpartum depression and was mailed to mothers at 1 month after delivery and returned within 4 months

\section{Child care environment}

The self-rating questionnaire of the Evaluation of Environmental Stimulation (EES) was used to evaluate the child care environment. Mothers were asked to answer the questionnaire in the 6-month assessment period for infant development. The EES was devised based on the Home Observation for Measurement of the Environment (HOME) [24] and the Home Screening Questionnaire (HSQ) [25] as adapted for the Japanese cultural and social contexts of the child care environment [26]. The EES is composed of 30 items comprising six subscales, including "human involvement" (varied involvement in daily life, scored 0-9), "responsiveness" (maternal response to the child, scored 0-2), "avoidance of restriction and punishment" (avoidance of neglect of infant, scored 0-1), "physical involvement" (appropriate maternal physical stimulus of the infant, scored 0-4), "social involvement" (opportunities for social interaction outside the home, scored 0-6), "organisation of the environment" (organisation of the physical environment, scored 0-3) and "social support" (social support in child rearing, scored 0-5). Higher scores indicate better child care environments.

\section{Statistical analysis}

A series of univariable and multivariable analyses was conducted using the following procedure. (1) In order to detect confounding variables that were possibly correlated to maternal depression during pregnancy, univariable analyses exploring correlation between the antenatal EPDS score and potential confounders (factors adjusted in previous studies, including characteristics of mothers, fathers, infants and child care environments) were carried out using a Spearman's correlation test, a Mann-Whitney $U$ test and a Kraskal-Wallis test; (2) the same nonparametric tests were conducted between BSID-II (MDI, PDI) scores and potential confounders to detect confounders that were possibly related to infant development; (3) univariable 
analyses using a Spearman's correlation test and a MannWhitney $U$ test were carried out to identify any correlation between maternal antenatal depression and infant development; (4) as a final justification, multivariable analyses entering the antenatal EPDS score as an independent variable and the MDI and PDI scores as outcome variables were conducted with and without adjusting for confounders, the results of which indicated a significant association of $p<0.01$ in steps (1) and (2) of the univariable analyses. In this final process, case-control comparison between depressed and non-depressed women during pregnancy was not possible because there were only nine $(5.8 \%)$ depressed women in this study. Therefore, we applied linear regression analyses using the total score of antenatal EPDS as a continuous variable, which minimised the influence of the low number of depressed women during pregnancy. The MDI and PDI scores were transformed into $\log 10$ scales because the distributions were skewed, while the independent variable of the antenatal EPDS score was hypothesised to follow a normal distribution according to the central limit theorem based on the sample size of over 100 [27].

Based on the results of analyses (1)-(4), gestational age and intrauterine growth restriction (IUGR) were thought to be significant confounders between depression during pregnancy and infant development. The correlation of the antenatal EPDS score with gestational age and IUGR was therefore analysed. Further linear regression analyses as well as logistic regression analyses were carried out, entering gestational age and IUGR as outcome variables and the antenatal EPDS score as an independent variable. We found no multicollinearity in a series of regression analyses. The goodness-of-fit for all regression models was evaluated by using adjusted $R^{2}$ and $F$ test.

\section{Informed consent and ethical review}

This study was conducted after obtaining written informed consent from all participants and was approved by the Institutional Ethics Board for epidemiologic studies at the Hokkaido University Graduate School of Medicine.

\section{Results}

Table 1 presents the characteristics of the mothers, fathers, infants and the child care environment. The mean ( \pm standard deviation, SD) maternal age at delivery was $31.4 \pm 4.9$ years. Twenty-six $(16.9 \%)$ mothers had a low annual household income ( $<3,000,000$ yen), 22 (14.3 $\%)$ mothers smoked during pregnancy and 60 (39.0\%) mothers reported stressful life events during the year before pregnancy. There were 78 male $(50.6 \%)$ and 76 female
(49.4\%) infants and 71 first-born infants $(46.1 \%)$. The mean $(+\mathrm{SD})$ gestational age was $275.7 \pm 8.5$ days, and the mean infant birth weight was $3090.5 \pm 361.1 \mathrm{~g}$. In total, five $(3.2 \%)$ and three $(1.9 \%)$, infants were preterm and small for gestational age (SGA), respectively, and three $(1.9 \%)$ and $12(7.8 \%)$ infants had a low birth weight or IUGR, respectively. None of the women assessed had diabetes during pregnancy; however, the cohort included 17 women with pregnancy-induced hypertension, seven with thyroid disease, and two with mental diseases, one of whom was prescribed a minor tranquilliser.

Table 2 presents data on antenatal and postnatal depression of mothers and data for infant development. The EPDS identified nine mothers with depression during pregnancy $(5.8 \%)$ and 21 mothers with depression after delivery $(13.6 \%)$. The median MDI and PDI scores were 90 [interquartile range (IQR, 25th-75th percentile) 88-94] and 88 (IQR 82-97), respectively.

Table 3 presents the results of the univariable analyses between the antenatal EPDS score, BSID-II (MDI, PDI) scores, and potential confounders. Potential confounding variables during pregnancy that showed significant association $(p<0.10)$ with the antenatal EPDS score were maternal education level $(p=0.055)$, household income $(p=0.076)$, past depressive symptoms $(p<0.000)$, worrying $(p<0.000)$, obsessiveness $(p<0.000)$, father's age $(r=-0.14, \quad p=0.088)$ and father's education level $(p=0.096)$. Postnatal EPDS was also found to be statistically significantly related to antenatal EPDS $(r=-0.48$, $p<0.000$ ) (Table 4). Potential confounding factors that were significantly associated with MDI included infant sex $(p=0.067)$, IUGR $(p=0.059)$, gestational age $(r=0.19$, $p=0.019)$, birth weight $(r=0.15, p=0.068)$, infant length $(r=0.15, p=0.067)$ and head circumference $(r=0.13, p=0.097)$. Potential confounding variables significantly related to PDI included caffeine intake during pregnancy $(r=-0.16, \quad p=0.043), \quad$ gestational age $(r=0.24, p=0.002)$, birth weight $(r=0.14, p=0.079)$, infant length $(r=0.14, p=0.079)$, age at 6-month assessment $(r=0.16, p=0.046)$ and "avoidance of restriction and punishment" $(r=0.18, p=0.025)$. Maternal smoking during pregnancy and maternal age, which were adjusted in previous studies, did not show statistical significance in correlation with antenatal EPDS, MDI or PDI.

Results of the univariable analyses for the MDI and PDI scores in relation to the antenatal and postnatal EPDS scores are shown in Table 4. Maternal antenatal EPDS tended to be significantly correlated to MDI $(r=-0.15$, $p=0.057)$, while there was no significant association between maternal postnatal depression and infant development.

We conducted linear regression analyses between the antenatal EPDS score and the MDI and PDI scores and 
Table 1 Characteristics of mothers, fathers, infants, and childcare environments

\begin{tabular}{|c|c|}
\hline Characteristic & Mean $\pm \mathrm{SD}, n(\%)$ \\
\hline \multicolumn{2}{|l|}{ Maternal characteristics } \\
\hline Age (years) & $31.4 \pm 4.9$ \\
\hline \multicolumn{2}{|l|}{ Education level (years) } \\
\hline$\leq 9$ & $5(3.2)$ \\
\hline $10-12$ & $54(35.1)$ \\
\hline $13-16$ & $92(59.7)$ \\
\hline$\geq 17$ & $3(1.9)$ \\
\hline \multicolumn{2}{|l|}{ Household income (yen/year) } \\
\hline$<3,000,000$ & $26(16.9)$ \\
\hline $3,000,000-5,000,000$ & $68(44.2)$ \\
\hline $5,000,000-7,000,000$ & $40(26.0)$ \\
\hline$>7,000,000$ & $20(13.0)$ \\
\hline Worked during pregnancy & $23(14.9)$ \\
\hline Smoked during pregnancy & $22(14.3)$ \\
\hline Caffeine intake during pregnancy (mg/day) & $123.4(80.2-183.1)^{\mathrm{a}}$ \\
\hline Alcohol intake during pregnancy (g/day) & $0.0(0.0-0.9)^{\mathrm{a}}$ \\
\hline Stressful life events before pregnancy & $60(39.0)$ \\
\hline \multicolumn{2}{|l|}{ Self-reported psychological status } \\
\hline Past depressive symptoms & $18(11.7)$ \\
\hline Worrying & $70(45.5)$ \\
\hline Obsessiveness & $45(29.2)$ \\
\hline \multicolumn{2}{|l|}{ Readiness for pregnancy } \\
\hline Planned pregnancy & $77(50.0)$ \\
\hline Wanted pregnancy & $131(85.1)$ \\
\hline Worked at 6 months postpartum & $17(11.0)$ \\
\hline \multicolumn{2}{|l|}{ Paternal characteristics } \\
\hline Age (years) & $33.2 \pm 5.8$ \\
\hline \multicolumn{2}{|l|}{ Education level (years) } \\
\hline$\leq 9$ & $4(2.6)$ \\
\hline $10-12$ & $53(34.4)$ \\
\hline $13-16$ & $80(51.9)$ \\
\hline$\geq 17$ & $17(11.0)$ \\
\hline \multicolumn{2}{|l|}{ Infant characteristics } \\
\hline Male & $78(50.6)$ \\
\hline First born (parity $=0$ ) & $71(46.1)$ \\
\hline Preterm birth & $5(3.2)$ \\
\hline SGA & $3(1.9)$ \\
\hline LBW & $3(1.9)$ \\
\hline IUGR & $12(7.8)$ \\
\hline Gestational age (days) & $275.7 \pm 8.5$ \\
\hline Birth weight (g) & $3,090.5 \pm 361.1$ \\
\hline Length $(\mathrm{cm})$ & $48.3 \pm 1.7$ \\
\hline Head circumference $(\mathrm{cm})$ & $33.3 \pm 1.3$ \\
\hline Chest circumference $(\mathrm{cm})$ & $31.5 \pm 1.4$ \\
\hline Age at 6-month assessment (days) & $190.3 \pm 8.7$ \\
\hline \multicolumn{2}{|l|}{ Child care environment } \\
\hline \multicolumn{2}{|l|}{ EES subscores at 6 months } \\
\hline Humanistic involvement & $7(7-8)^{\mathrm{a}}$ \\
\hline
\end{tabular}

Table 1 continued

\begin{tabular}{ll}
\hline Characteristic & Mean $\pm \mathrm{SD}, n(\%)$ \\
\hline Responsiveness & $2(2-2)^{\mathrm{a}}$ \\
Avoidance of restriction and punishment & $1(1-1)^{\mathrm{a}}$ \\
Physical involvement & $3(2-3)^{\mathrm{a}}$ \\
Social involvement & $4(3-5)^{\mathrm{a}}$ \\
Organisation of environment & $2(2-3)^{\mathrm{a}}$ \\
Social support & $5(4-5)^{\mathrm{a}}$ \\
\hline
\end{tabular}

EES Evaluation of environmental stimulation, IUGR intrauterine growth restriction, $L B W$ low birth weight, $S D$ standard deviation, $S G A$ small for gestational age

a These data are presented as the median with the interquartile range (IQR, 25th-75th percentile) given in parenthesis

Table 2 Antenatal and postnatal maternal depression and infant development

\begin{tabular}{|c|c|}
\hline Maternal depression/infant development & $\begin{array}{l}\text { Median (IQR), } \\
n(\%)\end{array}$ \\
\hline \multicolumn{2}{|l|}{ Maternal depression } \\
\hline \multicolumn{2}{|l|}{ Antenatal EPDS ${ }^{\mathrm{a}}$} \\
\hline Total score & $1(0-3)$ \\
\hline$\leq 8$ & $145(94.2)$ \\
\hline$\geq 9$ & $9(5.8)$ \\
\hline \multicolumn{2}{|l|}{ Postnatal EPDS ${ }^{b}$} \\
\hline Total score & $3(1-6)$ \\
\hline$\leq 8$ & $133(86.4)$ \\
\hline$\geq 9$ & $21(13.6)$ \\
\hline \multicolumn{2}{|l|}{ Infant development ${ }^{\mathrm{c}}$} \\
\hline BSID-II mental development index (MDI) & $90(88-94)$ \\
\hline BSID-II psychomotor development index (PDI) & $88(82-97)$ \\
\hline \multicolumn{2}{|c|}{$\begin{array}{l}\text { BSID-II Bayley Scales of Infant Development II, EPDS Edinburgh } \\
\text { Postnatal Depression Scale }\end{array}$} \\
\hline \multicolumn{2}{|c|}{$\begin{array}{l}\text { a Maternal depression between the second and the third trimesters } \\
\text { (23-35 gestational weeks) }\end{array}$} \\
\hline \multicolumn{2}{|l|}{ b Maternal depression after delivery (1-4 months) } \\
\hline $\begin{array}{l}\text { c Infant development at } 6 \text { months (from } 5 \text { month } \\
6 \text { months and } 15 \text { days after birth) }\end{array}$ & and $16 \mathrm{da}$ \\
\hline
\end{tabular}

adjusted for any factors with an association of $p<0.10$ in univariable analyses (Tables 5,6). Model 1 was adjusted for infant factors, namely, infant sex, IUGR, gestational age, birth weight, length, head circumference and age at 6-month assessment. Model 2 was adjusted using these same parameters as well as maternal caffeine intake during pregnancy and the child care factor "avoidance of restriction and punishment". Model 3 was a full model that adjusted for all covariants with a significant association of $p<0.10$ in the univariable analyses, namely, father's age 
Table 3 Maternal antenatal depression (EPDS) and infant development (BSID-II, MDI and PDI) in relation to potential confounding variables

\begin{tabular}{|c|c|c|c|c|c|c|c|}
\hline & \multirow[t]{2}{*}{$n$} & \multicolumn{2}{|c|}{ Antenatal EPDS ${ }^{\mathrm{a}}$} & \multicolumn{2}{|l|}{$\mathrm{MDI}^{\mathrm{b}}$} & \multicolumn{2}{|l|}{$\mathrm{PDI}^{\mathrm{b}}$} \\
\hline & & Mean \pm SD & $p$ & Mean \pm SD & $p$ & Mean \pm SD & $p$ \\
\hline \multicolumn{8}{|l|}{ Maternal characteristics } \\
\hline Age $(\text { years })^{c}$ & & $r=-0.11$ & 0.184 & $r=0.01$ & 0.867 & $r=0.01$ & 0.865 \\
\hline \multicolumn{8}{|l|}{ Education level (years) ${ }^{\mathrm{d}}$} \\
\hline$\leq 12$ & 55 & $3.07 \pm 3.70$ & 0.055 & $91.39 \pm 4.96$ & 0.147 & $90.80 \pm 10.95$ & 0.492 \\
\hline$\geq 13$ & 87 & $2.02 \pm 2.67$ & & $90.95 \pm 5.98$ & & $89.73 \pm 10.41$ & \\
\hline \multicolumn{8}{|l|}{ Household income (yen/year) ${ }^{\mathrm{e}}$} \\
\hline$<3,000,000$ & 26 & $3.85 \pm 3.87$ & 0.076 & $89.38 \pm 5.25$ & 0.415 & $89.15 \pm 11.18$ & 0.807 \\
\hline $3,000,000-5,000,000$ & 68 & $2.18 \pm 2.68$ & & $91.69 \pm 5.25$ & & $90.76 \pm 10.27$ & \\
\hline $5,000,000-7,000,000$ & 40 & $1.78 \pm 2.57$ & & $90.10 \pm 5.12$ & & $90.05 \pm 11.19$ & \\
\hline$>7,000,000$ & 20 & $2.42 \pm 3.14$ & & $91.45 \pm 7.16$ & & $89.45 \pm 10.42$ & \\
\hline \multicolumn{8}{|l|}{ Worked during pregnancy ${ }^{\mathrm{d}}$} \\
\hline No & 131 & $2.58 \pm 3.32$ & 0.342 & $90.63 \pm 5.37$ & 0.301 & $90.16 \pm 10.41$ & 0.754 \\
\hline Yes & 23 & $1.52 \pm 1.59$ & & $92.13 \pm 6.81$ & & $90.00 \pm 11.89$ & \\
\hline \multicolumn{8}{|l|}{ Smoked during pregnancy ${ }^{\mathrm{d}}$} \\
\hline No & 132 & $2.34 \pm 2.97$ & 0.913 & $90.83 \pm 5.64$ & 0.856 & $90.33 \pm 10.55$ & 0.619 \\
\hline Yes & 22 & $2.91 \pm 4.05$ & & $90.05 \pm 5.59$ & & $89.00 \pm 11.09$ & \\
\hline Caffeine intake during pregnancy $(\mathrm{mg} / \text { day })^{\mathrm{c}}$ & & $r=0.18$ & 0.827 & $r=-0.04$ & 0.662 & $r=-0.16$ & 0.043 \\
\hline Alcohol intake during pregnancy $(\mathrm{g} / \mathrm{day})^{\mathrm{c}}$ & & $r=0.11$ & 0.160 & $r=-0.07$ & 0.383 & $r=-0.04$ & 0.671 \\
\hline \multicolumn{8}{|l|}{ Stressful life events before pregnancy ${ }^{\mathrm{d}}$} \\
\hline No & 94 & $2.27 \pm 3.14$ & 0.220 & $91.14 \pm 5.22$ & 0.450 & $89.34 \pm 11.47$ & 0.162 \\
\hline Yes & 60 & $2.67 \pm 3.16$ & & $90.42 \pm 6.19$ & & $90.38 \pm 9.03$ & \\
\hline \multicolumn{8}{|l|}{ Self-reported psychological status } \\
\hline \multicolumn{8}{|l|}{ Past depressive symptoms ${ }^{\mathrm{d}}$} \\
\hline No & 136 & $1.96 \pm 2.58$ & $<0.001$ & $90.75 \pm 5.73$ & 0.337 & $90.17 \pm 10.68$ & 0.861 \\
\hline Yes & 18 & $5.94 \pm 4.56$ & & $91.67 \pm 4.67$ & & $89.89 \pm 10.31$ & \\
\hline \multicolumn{8}{|l|}{ Worrying $^{\mathrm{d}}$} \\
\hline No & 84 & $1.29 \pm 1.74$ & $<0.001$ & $91.21 \pm 5.67$ & 0.383 & $89.37 \pm 10.13$ & 0.387 \\
\hline Yes & 70 & $3.79 \pm 3.84$ & & $90.43 \pm 5.56$ & & $91.06 \pm 11.15$ & \\
\hline \multicolumn{8}{|l|}{ Obsessiveness $^{\mathrm{d}}$} \\
\hline No & 109 & $1.76 \pm 2.29$ & 0.001 & $90.66 \pm 5.85$ & 0.686 & $89.84 \pm 10.49$ & 0.556 \\
\hline Yes & 45 & $4.02 \pm 4.21$ & & $91.33 \pm 5.03$ & & $90.84 \pm 10.94$ & \\
\hline \multicolumn{8}{|l|}{ Readiness for pregnancy } \\
\hline \multicolumn{8}{|l|}{ Planned pregnancy ${ }^{\mathrm{d}}$} \\
\hline No & 77 & $2.66 \pm 3.44$ & 0.677 & $90.92 \pm 6.28$ & 0.880 & $90.12 \pm 11.52$ & 0.912 \\
\hline Yes & 77 & $2.18 \pm 2.81$ & & $90.79 \pm 4.90$ & & $90.16 \pm 9.68$ & \\
\hline \multicolumn{8}{|l|}{ Wanted pregnancy ${ }^{\mathrm{d}}$} \\
\hline No & 23 & $3.39 \pm 4.20$ & 0.238 & $91.65 \pm 6.78$ & 0.945 & $91.22 \pm 10.04$ & 0.548 \\
\hline Yes & 131 & $2.25 \pm 2.90$ & & $90.72 \pm 5.40$ & & $89.95 \pm 10.72$ & \\
\hline \multicolumn{8}{|l|}{ Worked at 6 months ${ }^{\mathrm{d}}$} \\
\hline No & 137 & $2.48 \pm 3.27$ & 0.988 & $90.92 \pm 5.62$ & 0.772 & $89.92 \pm 10.52$ & 0.504 \\
\hline Yes & 17 & $1.94 \pm 1.89$ & & $90.35 \pm 5.71$ & & $91.88 \pm 11.43$ & \\
\hline \multicolumn{8}{|l|}{ Paternal characteristics } \\
\hline Age $(\text { years })^{\mathrm{c}}$ & & $r=-0.14$ & 0.088 & $r=-0.02$ & 0.845 & $r=-0.09$ & 0.273 \\
\hline \multicolumn{8}{|l|}{ Education level (years) ${ }^{\mathrm{d}}$} \\
\hline$\leq 12$ & 57 & $2.79 \pm 2.21$ & 0.096 & $90.65 \pm 5.74$ & 0.891 & $91.19 \pm 9.62$ & 0.167 \\
\hline$\geq 13$ & 97 & $2.21 \pm 3.15$ & & $90.98 \pm 5.56$ & & $89.52 \pm 11.14$ & \\
\hline Infant characteristics & & & & & & & \\
\hline
\end{tabular}


Table 3 continued

\begin{tabular}{|c|c|c|c|c|c|c|c|}
\hline & \multirow[t]{2}{*}{$n$} & \multicolumn{2}{|c|}{ Antenatal EPDS ${ }^{\mathrm{a}}$} & \multicolumn{2}{|l|}{$\mathrm{MDI}^{\mathrm{b}}$} & \multicolumn{2}{|l|}{$\mathrm{PDI}^{\mathrm{b}}$} \\
\hline & & Mean \pm SD & $p$ & Mean \pm SD & $p$ & Mean \pm SD & $p$ \\
\hline \multicolumn{8}{|l|}{$\operatorname{Sex}^{\mathrm{d}}$} \\
\hline Male & 78 & $2.31 \pm 2.82$ & 0.617 & $91.72 \pm 5.71$ & 0.067 & $90.81 \pm 10.03$ & 0.337 \\
\hline Female & 76 & $2.54 \pm 3.45$ & & $89.97 \pm 5.61$ & & $89.45 \pm 11.12$ & \\
\hline \multicolumn{8}{|l|}{ Parity $^{\mathrm{d}}$} \\
\hline 0 & 71 & $2.61 \pm 3.49$ & 0.963 & $91.17 \pm 5.62$ & 0.725 & $89.82 \pm 10.99$ & 0.553 \\
\hline$\geq 1$ & 83 & $2.27 \pm 2.82$ & & $90.59 \pm 5.63$ & & $90.41 \pm 10.31$ & \\
\hline \multicolumn{8}{|l|}{ Preterm birth } \\
\hline No & 149 & $2.37 \pm 3.06$ & 0.725 & $90.87 \pm 5.67$ & 0.992 & $90.09 \pm 10.49$ & 0.890 \\
\hline Yes & 5 & $4.00 \pm 5.15$ & & $90.40 \pm 3.85$ & & $91.40 \pm 15.13$ & \\
\hline \multicolumn{8}{|l|}{$\mathrm{SGA}^{\mathrm{d}}$} \\
\hline No & 151 & $2.43 \pm 3.16$ & 0.957 & $90.88 \pm 5.66$ & 0.659 & $90.18 \pm 10.70$ & 0.803 \\
\hline Yes & 3 & $2.00 \pm 2.65$ & & $89.67 \pm 2.08$ & & $88.00 \pm 3.00$ & \\
\hline \multicolumn{8}{|l|}{$\mathrm{LBW}^{\mathrm{d}}$} \\
\hline No & 151 & $2.38 \pm 3.13$ & 0.165 & $90.83 \pm 5.63$ & 0.757 & $90.24 \pm 10.61$ & 0.351 \\
\hline Yes & 3 & $4.33 \pm 3.51$ & & $92.00 \pm 5.29$ & & $85.00 \pm 10.39$ & \\
\hline \multicolumn{8}{|l|}{ IUGR $^{\mathrm{d}}$} \\
\hline No & 142 & $2.45 \pm 1.42$ & 0.859 & $90.62 \pm 5.55$ & 0.059 & $90.32 \pm 10.84$ & 0.477 \\
\hline Yes & 12 & $2.08 \pm 2.28$ & & $93.67 \pm 5.77$ & & $88.00 \pm 7.12$ & \\
\hline Gestational age (days) ${ }^{\mathrm{c}}$ & & $r=0.22$ & 0.006 & $r=0.19$ & 0.019 & $r=0.24$ & 0.002 \\
\hline Birth weight $(\mathrm{g})^{\mathrm{c}}$ & & $r=0.06$ & 0.479 & $r=0.15$ & 0.068 & $r=0.14$ & 0.079 \\
\hline Length $(\mathrm{cm})^{\mathrm{c}}$ & & $r=0.15$ & 0.065 & $r=0.15$ & 0.067 & $r=0.14$ & 0.079 \\
\hline Head circumference $(\mathrm{cm})^{\mathrm{c}}$ & & $r=0.14$ & 0.094 & $r=0.13$ & 0.097 & $r=0.07$ & 0.365 \\
\hline Chest circumference $(\mathrm{cm})^{\mathrm{c}}$ & & $r=0.07$ & 0.391 & $r=0.13$ & 0.113 & $r=0.09$ & 0.294 \\
\hline Age at 6-month assessment (days) ${ }^{\mathrm{c}}$ & & $r=0.07$ & 0.385 & $r=0.09$ & 0.275 & $r=0.16$ & 0.046 \\
\hline \multicolumn{8}{|l|}{ Childcare environment } \\
\hline \multicolumn{8}{|l|}{ EES subscores at 6 months } \\
\hline Human involvement $^{\mathrm{c}}$ & & $r=0.20$ & 0.018 & $r=0.04$ & 0.610 & $r=-0.04$ & 0.958 \\
\hline Responsiveness $^{\mathrm{c}}$ & & $r=0.05$ & 0.530 & $r=0.10$ & 0.200 & $r=0.03$ & 0.705 \\
\hline Avoidance of restriction and punishment ${ }^{\mathrm{c}}$ & & $r=-0.56$ & 0.491 & $r=0.93$ & 0.252 & $r=0.18$ & 0.025 \\
\hline Physical involvement ${ }^{\mathrm{c}}$ & & $r=-0.11$ & 0.165 & $r=-0.04$ & 0.667 & $r=-0.09$ & 0.251 \\
\hline Social involvement ${ }^{\mathrm{c}}$ & & $r=0.20$ & 0.018 & $r=-0.03$ & 0.740 & $r=-0.13$ & 0.120 \\
\hline Organisation of environment ${ }^{\mathrm{c}}$ & & $r=0.10$ & 0.242 & $r=-0.07$ & 0.378 & $r=-0.02$ & 0.821 \\
\hline Social support ${ }^{\mathrm{c}}$ & & $r=-0.21$ & 0.011 & $r=-0.06$ & 0.474 & $r=0.03$ & 0.694 \\
\hline
\end{tabular}

Potential confounding variables including characteristics of mothers, fathers, infants, and childcare environments

EES evaluation of environmental stimulation, EPDS Edinburgh Postnatal Depression Scale, IUGR intrauterine growth restriction, $L B W$ low birth weight, $M D I$ mental development index, $P D I$ psychomotor development index, SGR small for gestational age

${ }^{\text {a }}$ Maternal antenatal depression between the second and the third trimesters (23-35 gestational weeks)

${ }^{\mathrm{b}}$ Infant mental and psychomotor development at 6 months (from 5 months and 16 days to 6 months and 15 days after birth); Statistical analyses

c Spearman correlation

d Mann-Whitney $U$ test

e Kruskal-Wallis test

and father's educational level in addition to factors adjusted for in Model 2. In the linear regression analyses, $p<0.05$ was considered to indicate a significant association.
Table 5 presents the MDI score in relation to the antenatal EPDS score and confounding variables based on the crude model (goodness of fit: adjusted $R^{2}=0.007$, $F=2.07, p=0.153$ ), model 1 (adjusted $R^{2}=0.087$, 
Table 4 Infant development (BSID-II, MDI and PDI) in relation to antenatal and postnatal maternal depression (EPDS)

\begin{tabular}{|c|c|c|c|c|c|c|c|}
\hline \multirow[t]{2}{*}{ Maternal characteristics } & \multirow[t]{2}{*}{$n$} & \multicolumn{2}{|c|}{ Antenatal EPDS ${ }^{\mathrm{a}}$} & \multicolumn{2}{|l|}{$\mathrm{MDI}^{\mathrm{b}}$} & \multicolumn{2}{|l|}{$\mathrm{PDI}^{\mathrm{b}}$} \\
\hline & & Mean $\pm \mathrm{SD}$ & $p$ & Mean $\pm \mathrm{SD}$ & $p$ & Mean $\pm \mathrm{SD}$ & $p$ \\
\hline \multicolumn{8}{|l|}{ Antenatal EPDS } \\
\hline Total score ${ }^{c}$ & & & & $r=-0.15$ & 0.057 & $r=-0.1$ & 0.881 \\
\hline$\leq 8^{\mathrm{d}}$ & 145 & & & $90.88 \pm 5.71$ & 0.756 & $90.06 \pm 10.51$ & 0.841 \\
\hline$\geq 9$ & 9 & & & $90.44 \pm 3.92$ & & $91.33 \pm 12.57$ & \\
\hline \multicolumn{8}{|c|}{ Postnatal EPDS at 1 month } \\
\hline Total score ${ }^{\mathrm{c}}$ & & $r=0.48$ & $<0.000$ & $r=-0.16$ & 0.679 & $r=-0.03$ & 0.216 \\
\hline$\leq 8^{\mathrm{d}}$ & 133 & $1.88 \pm 2.54$ & $<0.000$ & $90.96 \pm 5.80$ & 0.302 & $89.71 \pm 10.68$ & 0.798 \\
\hline$\geq 9$ & 21 & $5.86 \pm 4.30$ & & $90.19 \pm 4.33$ & & $92.81 \pm 9.94$ & \\
\hline
\end{tabular}

EPDS Edinburgh Postnatal Depression Scale, MDI Mental Development Index, PDI Psychomotor Development Index

${ }^{a}$ Maternal antenatal depression between the second and the third trimesters (23-35 gestational weeks)

b Infant mental and psychomotor development at 6 months (from 5 months and 16 days to 6 months and 15 days after birth); Statistical analyses c Spearman correlation

${ }^{\mathrm{d}}$ Mann-Whitney $U$ test

$F=2.81, p=0.006$ ), model 2 (adjusted $R^{2}=0.080$, $F=2.34, p=0.014$ ) and model 3 (adjusted $R^{2}=0.069$, $F=1.94, p=0.034)$. Even though the validity of all statistical models except the crude model was assured at the level of $p<0.05$, the adjusted $R^{2}$ was highest in model 1. A significant association between antenatal EPDS and MDI was not found in the crude model or in any of the adjusted models (crude: $\beta=-0.00,95 \%$ CI $[-0.00$, $0.00], p=0.153$; model $1: \beta=-0.05,95 \%$ CI $[-0.00$, $0.00], p=0.500$; model $2: \beta=-0.05,95 \%$ CI $[-0.00$, $0.00], p=0.552$; model $3: \beta=-0.05,95 \%$ CI $[-0.00$, $0.00], p=0.585$ ). To the contrary, gestational age showed a significant relation to MDI with consistently larger regression coefficients than those of the other factors even though the statistical model had been changed (model 1 : $\beta=0.23,95 \%$ CI $[0.00,0.00], p=0.013$; model 2 : $\beta=0.22,95 \%$ CI $[0.00,0.00], p=0.019$; model 3 : $\beta=0.23,95 \%$ CI $[0.00,0.00], p=0.018)$. Similarly, IUGR showed a significant relation to MDI in all models (model 1: $\beta=0.19,95 \%$ CI [0.00, 0.04], $p=0.020$; model 2: $\beta=0.21,95 \%$ CI $[-0.00,0.04], p=0.015$; model 3: $\beta=0.21,95 \%$ CI [0.00, 0.04], $p=0.017$ ).

Table 6 presents the PDI score in relation to the antenatal EPDS score and confounding variables based on the crude model (goodness of fit: adjusted $R^{2}=-0.007$, $F=0.01, p=0.927$ ), model 1 (adjusted $R^{2}=0.092$, $F=2.93, p=0.005$ ), model 2 (adjusted $R^{2}=0.133$, $F=3.34, p=0.001$ ) and model 3 (adjusted $R^{2}=0.141$, $F=3.09, p=0.001$ ). Each adjusted model was validated at the level of $p<0.01$; however, model 3 showed the highest value of adjusted $R^{2}$. While there was no significant correlation between antenatal EPDS and PDI in all models, PDI did show an association with gestational age (model 1: $\beta=0.28,95 \%$ CI $[0.00,0.00], p=0.003$; model 2 : $\beta=0.25,95 \%$ CI $[0.00,0.03], p=0.006$; model 3 : $\beta=0.23,95 \%$ CI $[0.00,0.00], p=0.012)$, infant age at the 6-month assessment (model 1: $\beta=0.25,95 \% \mathrm{CI}$ $[0.00,0.00], p=0.002$; model $2: \beta=0.24,95 \%$ CI [0.00, $0.00], p=0.003$; model $3: \beta=0.24,95 \%$ CI [0.00, 0.00$]$, $p=0.002)$, and "avoidance of restriction and punishment" (model 2: $\beta=0.20,95 \%$ CI [0.01, 0.07], $p=0.010$; model 3: $\beta=0.23,95 \%$ CI [0.02, 0.08], $p=0.004$ ).

Although we found no significant relation between antenatal EPDS and MDI or PDI in our linear regression analyses, the Spearman's correlation test detected a trend towards a correlation between antenatal EPDS and MDI $(r=-0.15, p=0.057)$ (Table 3$)$. To the contrary, antenatal EPDS was significantly associated with gestational age in Spearman's correlation $(r=0.22, \quad p=0.006)$ (Table 3) and, moreover, gestational age and IUGR were significantly related to MDI or PDI in the linear regression analyses. In order to explore the association between all of these variables in detail, we conducted further multiple linear regression analyses on gestational age and logistic regression analyses on IUGR in relation to antenatal EPDS (Table 7), adjusting for all potential confounders before delivery. The factors adjusted were: maternal factors (age, education level, household income, worked during pregnancy, smoked during pregnancy, caffeine intake during pregnancy, alcohol intake during pregnancy, stressful life events before pregnancy, past depressive symptoms, worrying, obsessiveness, planned pregnancy, wanted pregnancy), paternal factors (age and education level) and infant factors (sex and parity).

As a consequence, antenatal EPDS was significantly correlated to gestational age in the crude model 


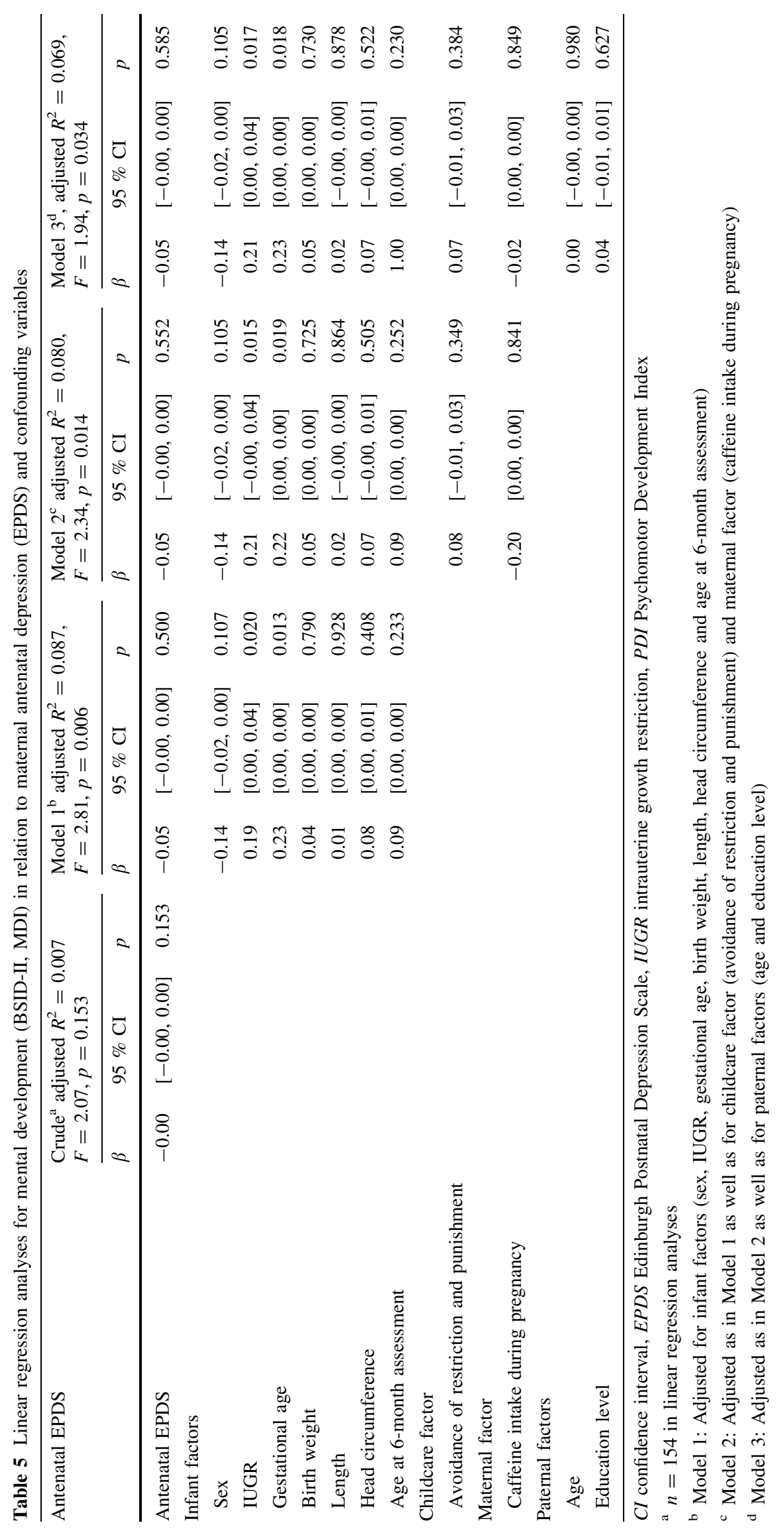




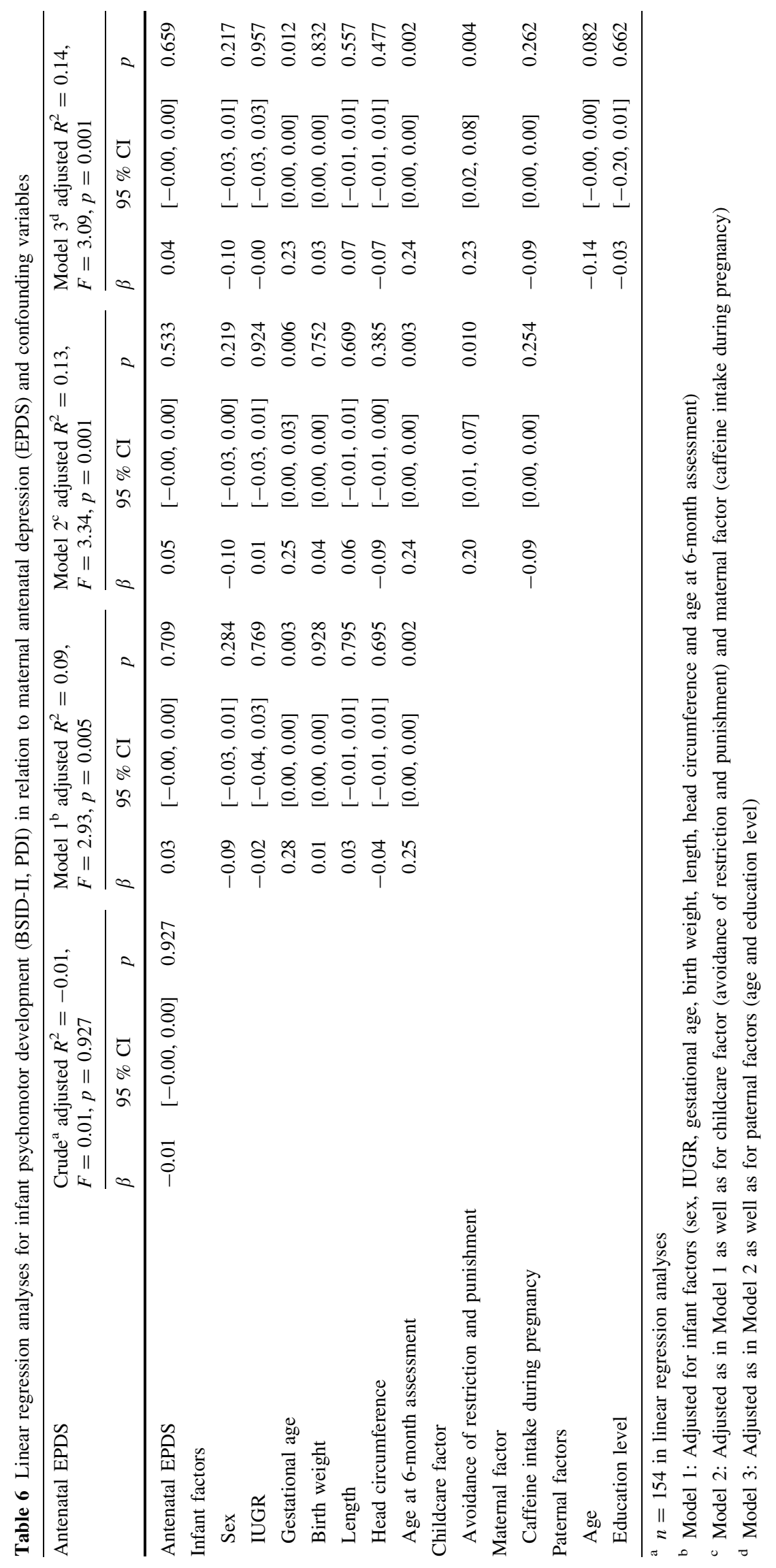


Table 7 Gestational age and IUGR in relation to maternal antenatal depression (EPDS) and confounding variables ${ }^{\mathrm{a}}$

\begin{tabular}{|c|c|c|c|c|c|c|}
\hline & \multicolumn{3}{|c|}{ Gestational age $^{\mathrm{b}}$} & \multicolumn{3}{|c|}{ IUGR $^{\mathrm{c}}$} \\
\hline & $\beta$ & $95 \% \mathrm{CI}$ & $p$ & OR & $95 \% \mathrm{CI}$ & $p$ \\
\hline Crude & \multicolumn{3}{|c|}{ Adjusted $R^{2}=0.03, F=5.07, p=0.026$} & \multicolumn{3}{|c|}{ Nagelkerke $R^{2}=0.003, \chi^{2}=0.16, p=0.686$} \\
\hline Antenatal EPDS & -0.18 & {$[-0.92,-0.06]$} & 0.026 & 0.96 & {$[0.78,1.18]$} & 0.697 \\
\hline Adjusted $^{\mathrm{d}}$ & \multicolumn{3}{|c|}{ Adjusted $R^{2}=0.12, F=2.19, p=0.006$} & \multicolumn{3}{|c|}{ Nagelkerke $R^{2}=0.27, \chi^{2}=18.24, p=0.571$} \\
\hline Antenatal EPDS & -0.25 & {$[-1.20,-0.17]$} & 0.010 & 0.81 & {$[0.59,1.11]$} & 0.199 \\
\hline \multicolumn{7}{|l|}{ Confounding variables } \\
\hline Stressful life events before pregnancy & -0.18 & {$[-5.91,-0.30]$} & 0.030 & 2.75 & {$[0.61,12.322]$} & 0.186 \\
\hline Planned pregnancy & 0.20 & {$[0.26,6.43]$} & 0.034 & 0.80 & {$[0.15,4.38]$} & 0.795 \\
\hline Infant sex; female & 0.17 & {$[0.02,5.57]$} & 0.048 & 0.43 & {$[0.08,2.38]$} & 0.333 \\
\hline First born & -0.23 & {$[-6.84,-1.11]$} & 0.007 & 1.14 & {$[0.23,5.77]$} & 0.875 \\
\hline
\end{tabular}

${ }^{a}$ Factors shown in this table were found to be statistically significant in relation to gestational age or IUGR

b Multiple linear regression analyses

c Logistic regression analyses; $n=154$

d Adjusted for maternal factors (age, education level, household income, worked during pregnancy, smoked during pregnancy, caffeine intake during pregnancy, alcohol intake during pregnancy, stressful life events before pregnancy, past depressive symptoms, worrying, obsessiveness, planned pregnancy, wanted pregnancy), paternal factors (age and education level) and infant factors (sex and parity)

$(\beta=-0.18,95 \%$ CI $[-0.92,-0.06], p=0.026$; goodness of fit: adjusted $\left.R^{2}=0.026, F=5.07, p=0.026\right)$ bit not to IUGR $(\mathrm{OR}=0.96,95 \% \mathrm{CI}[0.78,1.19], p=0.697$; goodness of fit: Nagelkerke $R^{2}=0.003, \chi^{2}=0.16$, $p=0.686)$. This trend did not change even when the confounders were adjusted. In the adjusted model analysing the association between antenatal EPDS and gestational age, the regression coefficient of antenatal EPDS was the highest of all variables $(\beta=-0.25,95 \%$ CI $[-1.20$, -0.17 ], $p=0.010$; goodness of fit: adjusted $R^{2}=0.123$, $F=2.19, p<0.000)$.

\section{Discussion}

Summary of study findings

In this study, we evaluated the hypothesis that maternal depression during pregnancy has an adverse relationship with infant development using improved adjustments for confounding variables. Although a trend of association between maternal antenatal depression and infant development was found in the univariable analysis, this correlation was lost in the multivariable analyses. However, the results of the regression analyses highlighted the fact that depression during pregnancy was significantly related to shorter gestational age and that shorter gestational age was significantly related to developmental delay in infant cognitive function. Therefore, gestational age can be considered to be an important confounder in the association between maternal antenatal depression and infant mental development. This is the first study to investigate the relationship between maternal depression during pregnancy and infant development with a proper control for gestational age, and the results thus provide new insights into the seemingly inconsistent results from previous studies.

Prevalence of maternal depression and scoring of infant development

The prevalence of maternal depression during pregnancy, defined using a cut-off of $8 / 9$ on the EPDS, was $5.8 \%$ in our study, which is relatively low compared with rates reported from Europe and the USA. Previous studies evaluating maternal depression during the second or third trimester reported prevalence levels of $7.0 \%$ in the USA [6], $13.9 \%$ in England [28], and $17.4 \%$ in Sweden [29] using the EPDS, and $8.7 \%$ in Hong Kong using the Beck Depression Scale [30]. However, the prevalence of depression during pregnancy based on the DSM-III-R Major Depressive Episode in Japan was reported to be $5.6 \%$ [31]. In addition, according to a meta-analysis of perinatal depression in developed countries [32], the prevalence of major and minor depression during pregnancy ranges from 6.5 to $12.9 \%$ (with minor depression ranging from 1.0 to $12.9 \%$ ); maternal depression at 1-2 months after delivery was estimated to be 10-15\%. The prevalence of antenatal and postnatal maternal depression in our study was within those ranges, thereby supporting the credibility of our results.

We used the BSID-II score to evaluate infant development in our study. The median MDI and PDI scores were 90 and 88 , respectively, and both are lower than standardised scores (mean score 100). Since both cultural and language 
differences exist between Japan and the USA, the BSID-II must be used with care in Japan. However, the first BSID edition has been used in Japan for the developmental assessment of infants [33], and a high correlation was reported between BSID-II and the Kyoto Developmental Test, which is standardised in Japan. Furthermore, a study in Taiwan revealed high reproducibility using BSID-II despite cultural differences [34]. To improve the reliability of the BSID-II scores in our study, we limited our evaluation of development to 6-month-old infants, and every examiner scored each infant. Therefore, the BSID-II scores of the participants in this study are directly comparable with each other.

Antenatal depression, gestational age and infant mental development

In previous studies examining the association of antenatal depression with infant development, Deave et al. [6] used the EPDS and reported that antenatal depression has an adverse impact on infant development, whereas DiPietro et al. [11]. used the BSID-II and reported a positive impact. Surprisingly, our results are inconsistent with both of these studies and successfully added new findings to current knowledge. There are several possible reasons why our results differ from those reported previously, especially in terms of controlling for confounders. First, our study differed from these previous studies with respect to the confounding factors entered into the statistical analyses and the credibility of this information. Although many potential confounders were considered by Deave et al. [6], all but four (antenatal tobacco use, maternal age, postnatal life events, postnatal depression) were removed using a conceptual framework. DiPietro et al. [11] also removed the gestational age confounder in the final analyses. Moreover, DiPietro et al. do not describe how information on gestational age was obtained. In our study, all perinatal information was obtained from medical records, ensuring the reliability of the data. Second, there are differences in childcare factors and in infant age at assessment. In our study, the child care environment was taken into account as a considerable confounder. In addition, infant development was evaluated at 6 months to minimise the influence of other confounding factors after birth. In the Deave et al. [6] and DiPietro et al. [11] studies, child assessments were conducted much later (18 and 24 months, respectively), and childcare factors were not controlled for throughout all the steps of the analysis. DiPietro et al. [11] reported a high level of maternal education (median 17 years), but Deave et al. [6] provided no information on maternal education. Higher education levels can counteract various negative influences that occur during the perinatal period [35]. It is also likely that other aspects of the child care environment positively or negatively affect infant development [36]. In our study, the education levels of both parents were also analysed statistically as confounding variables. Third, there were differences in the measures used. Deave et al. [6] applied the DDST, which evaluates similar developmental abilities as the BSID-II, but depends on parental reporting. Depressed mothers may possibly perceive their children's abilities as being lower, and such a reporting bias would lead to the apparent statistical association between antenatal depression and child development in these author's study. In contrast, reporting bias in infant assessment was avoided in our study by using an objective and blinded assessment, which provided improved credibility.

We identified gestational age to be a considerable confounding variable; that is, infants of depressed mothers tended to be delivered earlier and to suffer cognitive developmental delays as a consequence. A higher EPDS score during pregnancy was related to shorter gestational age $(\beta=-0.25,95 \%$ CI $[-1.20,-0.17], p=0.010)$, and shorter gestational age was related to lower scores of mental $(\beta=0.23,95 \%$ CI $[0.00,0.00], p=0.013)$ as well as psychomotor $(\beta=0.23,95 \%$ CI $[0.00,0.00], p=0.012)$ development in the adjusted linear regression analysis (Tables 5, 6). This finding supports the notion that the developmental delays were a consequence of early delivery brought on by maternal antenatal depression. Results from several studies substantiate the impact of maternal depression on the length of gestation by showing that antenatal depression is associated with either a reduced gestational age [37] or a greater incidence of preterm birth among severely depressed women compared to non-depressed women [38, 39]. The influence of antenatal depression on gestational age may be explained by a potential biological pathway. According to recent studies, cortisol increases the release of a placental corticotropin-releasing hormone (CRH) [39, 40], which plays a key role in triggering parturition [40-43]. Antenatal depression is related to a greater incidence of premature delivery and to elevated antenatal cortisol levels compared to non-depressed women [38]. Higher levels of cortisol and $\mathrm{CRH}$ have also been detected in women who delivered preterm infants compared with those who delivered at term [40]. In our study, it was not possible to analyse the relation between preterm birth and maternal depression during pregnancy due to only five women $(3.2 \%)$ in our cohort giving birth to preterm infants. However, it is important to explore the association between gestational age, preterm birth and maternal antenatal depression in greater detail in further studies.

\section{Child care factors and infant psychomotor development}

Infant PDI was related not only to gestational age but also to "avoidance of restriction and punishment" in this study. The positive association of "avoidance of restriction and 
punishment" with PDI in our study agrees with the results of earlier studies. Some studies have reported that infants exposed to maltreatment have lower PDI scores than control groups [44, 45], confirming our findings. However, the authors of these studies also noted that maltreatment had an impact on the MDI scores [44, 45], which we did not see. The reason for this difference may be the difference in infant age at the time of assessment. Previous studies examined infants aged between 2 and 30 months, whereas we enrolled infants aged 6 months in our study. In one earlier study, the reported impact of maltreatment on MDI appeared only after 14 months of age [46]; thus, the lack of association at an earlier age is consistent with our results.

\section{Study strengths and limitations}

This study constitutes a prospective cohort study, which minimises recall bias. We collected infant development scores through constructed assessment by examiners blinded to other data, enabling us to control for reporting bias and observer bias. We also collected perinatal information on mothers and infants (such as disease history, pregnancy conditions, and birth weight and size) from medical records written by obstetricians, not from maternal reports, which further increased data reliability. Moreover, diverse confounding variables were controlled for during a series of statistical analyses.

Nonetheless, this study has the following limitations. First, our sample size was relatively small to represent the general population, even though it was larger than those in several previous studies [10-12]. However, despite the small sample size, the goodness of fit in all of the adjusted models of linear regression analyses indicated statistical significance $(p<0.05)$, thereby endorsing the validity of the study results. Second, our study may have suffered from selection bias as it was based on a cohort from one regional hospital treating pregnant women in Sapporo and the surrounding areas, and the participant rate was low (30\%). Since women who were not interested in or were unwilling to participate in our study were excluded from the study, there is a possibility that depressed women may have been less likely to be involved in this study. The follow-up rate was also slightly low in our cohort study (50\%). Of 298 women with a single birth (96\% of all study participants), those who did not complete or return the mailed EPDS questionnaire between 1 and 4 months after delivery (10\% of those of single birth) and those who did not attend the infant assessment during the period from 5 months and 16 days up to 6 months and 15 days after birth (42\% of those who were assessed the postnatal EPDS) were excluded from the study. Because the BSID-II has not been standardised for use in Japan, we strictly limited the period of assessment, which may have resulted in the low follow- up rate. In our study, the prevalence of SGA was also very small $(1.9 \%)$. Pregnant women may possibly have avoided participating in our cohort study or dropped out during the follow-up period because of depression itself, causing a selection bias that may slightly lower the prevalence of depressive symptoms, as well as that of SGA. These factors may limit the extrapolation of our results to the general population. However, there was no remarkable difference between the prevalence of antenatal depression in all participants at the beginning (309 women, $5.2 \%$ ) and that in the analysed women (154 women, $5.8 \%$ ); therefore, we conclude that the low follow-up rate was unlikely to have had a significant influence on the study results. Finally, information on antenatal psychological distress may have been insufficient; maternal depression was not based on clinical diagnosis, and the experience of stressful events and the other maternal psychological factors were collected using dichotomous questions based on unstandardised questionnaires. However, the EPDS is thought to be a wellvalidated scale and was used in the previous study by Deave et al. [6] in the absence of clinical diagnosis.

In conclusion, the results of our study suggest that delay in infant mental development may be related to a shorter gestational period resulting from maternal depression during pregnancy. Because impaired cognitive and motor functions present at 6 months can be reversed by school age, further follow-up monitoring should continue at least until school age, and additional studies are required to clarify this issue.

Acknowledgements This study was supported by a Grant-in-Aid for Health Scientific Research from the Japan Ministry of Health, Labour and Welfare, and by a Grant-in-Aid for Scientific Research from the Japan Society for the Promotion of Science. We express our extreme gratitude to all families who took part in this study, to the obstetricians and nurses who helped recruit the patients and who provided medical data, and to the occupational therapists who performed the infant assessments.

Conflict of interest There are no conflicts of interest, including competing financial interest and financial relationship with the funding organizations, with regard to this manuscript.

\section{References}

1. Fameli M, Kitraki E, Stylianopoulou F. Effects of hyperactivity of the maternal hypothalamic-pituitary-adrenal (HPA) axis during pregnancy on the development of the HPA axis and brain monoamines of the offspring. Int J Dev Neurosci. 1994;12(7): $651-9$.

2. Welberg LA, Seckl JR. Prenatal stress, glucocorticoids and the programming of the brain. J Neuroendocrinol. 2001;13(2): 113-28.

3. Teixeira JM, Fisk NM, Glover V. Association between maternal anxiety in pregnancy and increased uterine artery resistance index: cohort based study. BMJ. 1999;318(7177):153-7. 
4. Weinstock M. Alterations induced by gestational stress in brain morphology and behaviour of the offspring. Prog Neurobiol. 2001;65(5):427-51.

5. Wadhwa PD. Psychoneuroendocrine processes in human pregnancy influence fetal development and health. Psychoneuroendocrinology. 2005;30(8):724-43.

6. Deave T, Heron J, Evans J, Emond A. The impact of maternal depression in pregnancy on early child development. BJOG. 2008;115(8):1043-51.

7. O'Connor TG, Heron J, Glover V. Antenatal anxiety predicts child behavioral/emotional problems independently of postnatal depression. J Am Acad Child Adolesc Psychiatry. 2002;41(12): 1470-7.

8. Laplante DP, Brunet A, Schmitz N, Ciampi A, King S. Project ice storm: prenatal maternal stress affects cognitive and linguistic functioning in 5 1/2-year-old children. J Am Acad Child Adolesc Psychiatry. 2008;47(9):1063-72.

9. Laplante DP, Barr RG, Brunet A. Galbaud du Fort G, Meaney ML, Saucier JF, et al. Stress during pregnancy affects general intellectual and language functioning in human toddlers. Pediatr Res. 2004;56(3):400-10.

10. Sugawara M, Kitamura T, Aoki M, Shima S. Maternal depression during pregnancy and newborn's behavioral characteristics. J Child Health. 1988;47(5):577-81.

11. DiPietro JA. Novak MF, Costigan KA, Atella LD, Reusing SP. Maternal psychological distress during pregnancy in relation to child development at age two. Child Dev. 2006;77(3):573-87.

12. Kaplan LA, Evans L, Monk C. Effects of mothers' prenatal psychiatric status and postnatal caregiving on infant biobehavioral regulation: can prenatal programming be modified? Early Hum Dev. 2008;84(4):249-56.

13. Mash EJ, Johnston C. A comparison of the mother-child interactions of younger and older hyperactive and normal children. Child Dev. 1982;53(5):1371-81.

14. Gardner FE. The quality of joint activity between mothers and their children with behaviour problems. J Child Psychol Psychiatry. 1994;35(5):935-48.

15. Famularo R, Kinscherff R, Fenton T. Psychiatric diagnoses of maltreated children: preliminary findings. J Am Acad Child Adolesc Psychiatry. 1992;31(5):863-7.

16. Nagata C, Kabuto M, Shimizu H. Association of coffee, green tea, and caffeine intakes with serum concentrations of estradiol and sex hormone-binding globulin in premenopausal Japanese women. Nutr Cancer. 1998;30:21-4.

17. Nagata C, Kabuto M, Takatsuka N, Shimizu H. Associations of alcohol, height, and reproductive factors with serum hormone concentrations in postmenopausal Japanese women. Steroid hormones in Japanese postmenopausal women. Breast Cancer Res Treat. 1997;44(3):235-41.

18. Cox JL, Holden JM, Sagovsky R. Detection of postnatal depression. Development of the 10-item Edinburgh Postnatal Depression Scale. Br J Psychiatry. 1987;150:782-6.

19. Okano T, Murata M, Masuji F, Tamaki R, Nomura J, Miyaoka H, et al. Validation and reliability of Japanese version of EPDS (Edinburgh Postnatal Depression Scale). Arch Psychiatr Diagnosis Clin Eval. 1996;7(4):525-33.

20. Bayley N. Manual for the Bayley scales of infant development. 2nd ed. New York: Psychological Corporation; 1993.

21. Nakajima S, Saijo Y, Kato S, Sasaki S, Uno A, Kanagami N, et al. Effects of prenatal exposure to polychlorinated biphenyls and dioxins on mental and motor development in Japanese children at 6 months of age. Environ Health Perspect. 2006;114(5):773-8.

22. Nakamura Y, Nakajima S, Yanagiya S, Sengoku Y, Tachi N, Kishi R. Developmental assessment for 6 months infants by the Bayley scales of infant development. 2nd edn. Part III: A validation study by using JDDST. J Jpn Occup Therap Assoc 2004;23: 392.

23. Nakajima S, Nakamura Y, Yanagiya S, Sengoku Y, Tachi N, Kishi R. Developmental assessment for 6 months infants by the Bayley scales of infant development. 2nd edn. Part I: Results of mental and psychomotor development. J Jpn Occup Therap Assoc 2004;23:390.

24. Bradley RH, Caldwell BM. Home observation for measurement of the environment: a validation study of screening efficiency. Am J Ment Defic. 1977;81(5):417-20.

25. Frankenburg WK, Coons CE. Home Screening Questionnaire: its validity in assessing home environment. J Pediatr. 1986;108(4): 624-6.

26. Anme T. Child support and evaluation and child care environment in the era of low birthrates. Tokyo: Kawashima Shoten; 1996.

27. Katz Mitchell H. Multivariable analysis: a practical guide for clinicians. 2nd ed. Cambridge: Cambridge University Press; 2006.

28. Evans J, Heron J, Francomb H, Oke S, Golding J. Cohort study of depressed mood during pregnancy and after childbirth. BMJ. 2001;323(7307):257-60.

29. Josefsson A, Berg G, Nordin C, Sydsjö G. Prevalence of depressive symptoms in late pregnancy and postpartum. Acta Obstet Gynecol Scand. 2001;80(3):251-5.

30. Chung TK, Lau TK, Yip AS, Chiu HF, Lee DT. Antepartum depressive symptomatology is associated with adverse obstetric and neonatal outcomes. Psychosom Med. 2001;63(5):830-4.

31. Kitamura T, Yoshida K, Okano T, Kinoshita K, Hayashi M, Toyoda N, et al. Multicentre prospective study of perinatal depression in Japan: incidence and correlates of antenatal and postnatal depression. Arch Womens Ment Health. 2006;9(3): 121-30.

32. Gavin NI, Gaynes BN, Lohr KN, Meltzer-Brody S, Gartlehner G, Swinson T. Perinatal depression: a systematic review of prevalence and incidence. Obstet Gynecol. 2005;106(5 Pt 1):1071-83.

33. Oka T, Suzuki K, Nakai K, Hosokawa T, Satoh H. A trial to apply Bayley Scales of Infant Development second edition to Japanese children. J Clin Exp Med. 2005;212:259-63.

34. Huang HL, Chuang SF, Jong YJ, Yu L, Shieh YL. Applicability of BSID-II in diagnosing developmental delay at Kaohsiung area. Kaohsiung J Med Sci. 2000;16:197-202.

35. Wang LW, Wang ST, Huang CC. Preterm infants of educated mothers have better outcome. Acta Paediatr. 2008;97(5):568-73.

36. Weisglas-Kuperus N, Baerts W, Smrkovsky M, Sauer PJ. Effects of biological and social factors on the cognitive development of very low birth weight children. Pediatrics. 1993;92(5):658-65.

37. Van Dijk AE, Van Eijsden M, Stronks K, Gemke RJ, Vrijkotte TG. Maternal depressive symptoms, serum folate status, and pregnancy outcome: results of the Amsterdam Born Children and their Development study. Am J Obstet Gynecol. 2010;203(6): 563-7.

38. Diego MA, Field T, Hernandez-Reif M, Schanberg S, Kuhn C, Gonzalez-Quintero VH. Prenatal depression restricts fetal growth. Early Hum Dev. 2009;85(1):65-70.

39. Dayan J, Creveuil C, Marks MN, Conroy S, Herlicoviez M, Dreyfus M, et al. Prenatal depression, prenatal anxiety, and spontaneous preterm birth: a prospective cohort study among women with early and regular care. Psychosom Med. 2006;68(6):938-46.

40. Sandman CA, Glynn L, Schetter CD, Wadhwa P, Garite T, Chicz-DeMet A, et al. Elevated maternal cortisol early in pregnancy predicts third trimester levels of placental corticotropin releasing hormone $(\mathrm{CRH})$ : priming the placental clock. Peptides. 2006;27(6):1457-63. 
41. Majzoub JA, Karalis KP. Placental corticotropin-releasing hormone: function and regulation. Am J Obstet Gynecol. 1999;180(1 Pt 3):S242-6.

42. Pike IL. Maternal stress and fetal responses: evolutionary perspectives on preterm delivery. Am J Hum Biol. 2005;17(1): $55-65$.

43. McLean M, Bisits A, Davies J, Woods R, Lowry P, Smith R. A placental clock controlling the length of human pregnancy. Nat Med. 1995;1(5):460-3.
44. Appelbaum AS. Developmental retardation in infants as a concomitant of physical child abuse. J Abnorm Child Psychol. 1977;5(4):417-23.

45. Koski MA, Ingram EM. Child abuse and neglect: effect on Bayley Scale scores. J Abnorm Child Psychol. 1977;5(1):79-91.

46. Allen R, Wasserman GA. Origins of language delay in abused infants. Child Abuse Negl. 1985;9(3):335-40. 